\title{
Milchallergie schrittweise ausgetrickst
}

Viele Kleinkinder reagieren auf Milchprodukte allergisch. Aber Kinder können sich an Milch gewöhnen, selbst wenn sie anfangs sensibel reagieren. Der Trick besteht darin, den Kindern zunächst Produkte anzubieten, in denen die enthaltene Milch sehr stark erhitzt (denaturiert) wurde. Später kann dann auf Nahrungsmittel mit weniger denaturierter Milch gewechselt werden

E twa $75 \%$ aller Kinder mit Kuhmilchallergie vertragen Backwaren, in denen Milch verarbeitet wurde, ohne Probleme. Ob man mit der regelmäßigen Verabreichung solcher Backwaren langfristig eine Art Desensibilisierung einleiten kann, untersuchten Wissenschaftler der Mount Sinai School of Medicine in New York bei 88 Kindern mit einer bestätigten Kuhmilchallergie.

Die kleinen Patienten mussten zunächst einen mit Kondensmilch hergestellten Muffin essen. 65 der 88 Patienten vertrugen dieses Gebäck problemlos. Die Eltern dieser Kinder wurden angehalten, den Milchanteil in der Nahrung mithilfe von Muffins kontinuierlich zu erhöhen.

Nach sechs bis zwölf Monaten stellten sich die „Muffintest-negativen“ Kinder in der Klinik nochmals vor. Diesmal erhielten sie eine Käsepizza mit weniger denaturierten Milchproteinen. 78\% der Kinder zeigten dabei keine Unverträglichkeitsreaktionen. Ihnen wurde geraten, künftig diesen Käse auf den Speiseplan zu setzen.

Diejenigen Kinder, die die Pizza nicht vertrugen, aßen weiterhin nur Backwaren mit Milch und kehrten nach weiteren sechs bis zwölf Monaten in die Klinik zurück, um sich erneut einem Test mit Pizza zu unterziehen. Zeigten sie dann keine Reaktionen, durften sie ebenfalls in Pizza enthaltenen Käse essen.

Nach drei Jahren wurden die Studienteilnehmer, die die Pizza vertragen hatten, erneut einbestellt. Unter Beobachtung durften sie nun Milch trinken, Eis oder Joghurt essen. Von den 65 Kindern zeigten dabei $60 \%$ auch gegen diese Lebensmittel keine Unverträglichkeitsreaktionen.

Gegen Kuhmilch sind Kleinkinder häufig sensibilisiert
Fazit: Zwar gehen Nahrungsmittelintoleranzen auch von selbst zurück - dies soll aber nur auf $22 \%$ der Patienten zutreffen. Die vorliegende Studie zeigt, dass mit einer guten ärztlichen Überwachung bei Kindern Unverträglichkeitsreaktionen gegen Nahrungsmittel viel schneller überwunden werden können.

Prof. Dr. Kurt Malberg

Kim JS et al. Dietary baked milk accelerates the resolution of cow's milk allergy in children. J Allergy and Clin Immunol 2011; 128 : 125-131

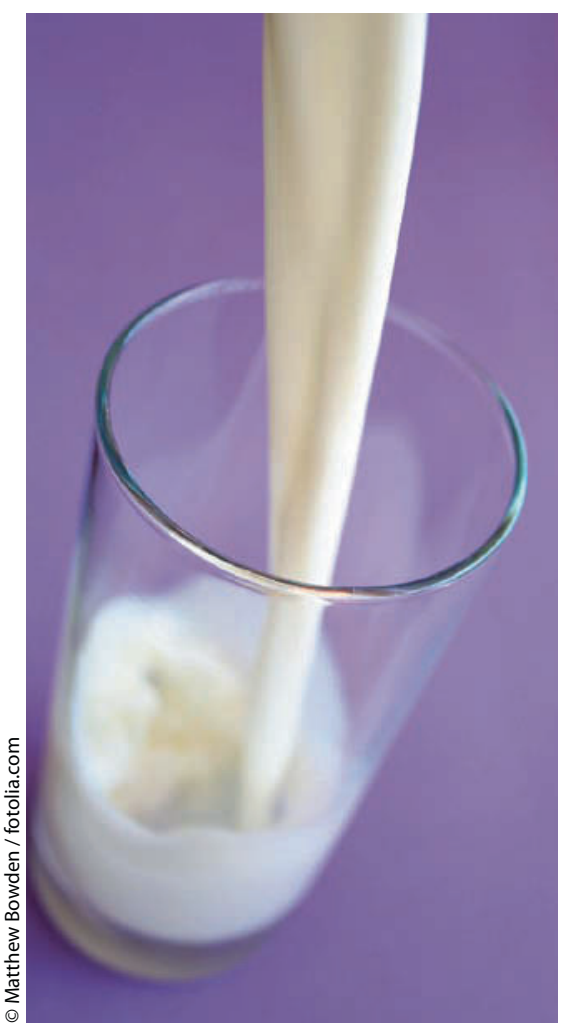

